EESTI NSV TEADUSTE AKADEEMIA TOIMETISED. 28. KOIDE KEEMIA. 1979, NR. 4

ИЗВЕСТИЯ АКАДЕМИИ НАУК ЭСТОНСКОП ССР. ТОМ 28 ХИМИЯ. 1979, № 4

удК $677.4: 543.226$

O. KIRRET, H. ZAHN, R. HACK

\title{
DIE THERMOANALYSE (DTA, TG) VON FLAMMWIDRIGEN UND FLAMMFESTAUSGERUSTETEN CHEMIEFASERN
}

Mit dem starken Eindringen der synthetischen Chemiefasern in das Textilgebiet gewann das Problem des Flammschutzes für diesen Fasersektor schnell an Bedeutung. Um die Flammwidrigkeit zu steigern, wurde zuerst hochtemperaturbeständige aromatische Polyamidfaser Nomex synthetisiert. Durch Polykondensation von 1,3-Phenylendiamin mit Isophtalsäure entsteht ein aromatisches Polyamid. Nomex ist nicht nur hervorragend chemisch widerstandsfähig, sondern verträgt Temperaturen bei $175^{\circ} \mathrm{C}$ bis 1000 St. ohne nennenswerten Festigkeitsverlust. Nomex ist schwer entflammbar, zersetzt sich bei 370 und verkohlt bei $400{ }^{\circ} \mathrm{C}$ (nicht schmelzend).

Kevlar ist eine Polyaramidfaser (Poly-1,4-phenylenterephtalamid), ein aromatisches Polyamid mit hoher Wärmefestigkeit $(85 \%$ der Normalfestigkeit nach $48 \mathrm{~h}$ Einwirkung von $\left.175^{\circ} \mathrm{C}\right)$. Kevlar ist schwer entflammbar.

Qiana ist ein alicyclisches Polyamid aus der Dodecandisäure mit 4,4-Diamino-dicyclohexylmethan polykondensiert. Qiana ist nicht besonders flammwidrig [ ${ }^{1}$ ].

Um Entflammbarkeit zu steigern, werden thermostabile Faserstoffe aus anorganischen und organischen Polymeren hergestellt, wobei verschiedene Flammschutzmittel, wie Metalloxide, Metallchloride, phosphororganische, Phosphorhalogen-, Phosphorstickstoffverbindungen usw. als Zusätze verwendet werden.

Für die technischen Zwecke werden Glas-, Kohlenstoff- und Graphitfasern sowie keramische Faser verwendet.

Elementaranalysen von Chemiefasern (C, H, N)

\begin{tabular}{|c|c|c|c|c|}
\hline \multirow{2}{*}{ Faserstoff } & \multicolumn{3}{|c|}{ Elementaranalysen \% } & \multirow{2}{*}{$100-(\mathrm{H}+\mathrm{C}+\mathrm{N})$} \\
\hline & $\mathrm{N}$ & C & $\mathrm{H}$ & \\
\hline $\begin{array}{l}\text { Nomex T } 450 \text { Du Pont } \\
\text { Aromatische Polyamidfaser }\end{array}$ & 11,40 & 68,15 & 4,59 & 15,86 \\
\hline $\begin{array}{l}\text { Kevlar Du Pont } \\
\text { Aromatische Polyaramidfaser (Poly-p- } \\
\text { phenylenterephtalamid) }\end{array}$ & 11,62 & 70,14 & 4,60 & 13,64 \\
\hline $\begin{array}{l}\text { Qiana Du Pont } \\
\text { Alicyclische Polyamidfaser }\end{array}$ & 6,59 & 71,78 & 10,83 & 10,80 \\
\hline $\begin{array}{l}\text { Orlon Type } 775 \text { Du Pont } \\
\text { Flammfeste Polyacrylnitrilfaser }\end{array}$ & 15,10 & 55,42 & 5,79 & 23,69 \\
\hline $\begin{array}{l}\text { Verel Eastman DDIF } \\
\text { Modifizierte Polyacrylnitrilfaser }\end{array}$ & 12,26 & 48,96 & 4,70 & 34,00 \\
\hline Modacrylic SEF Monsanto & 15,32 & 51,24 & 4,60 & 28,84 \\
\hline
\end{tabular}

Modifizierte Polyacrylnitrilfaser (selbstentlöschend) 
In unserer Arbeit wurden thermostabilisierte und flammfestausgerüstete aromatische Polyamidfasern (Nomex, Kevlar), alicyclische Polyamidfaser (Qiana), flammfeste Polyacrylnitrilfaser (Orlon Type 775 Du Pont), selbstentlöschende Verel- und Modacrylfaser untersucht. In der Tabelle sind die Elementaranalysen der untersuchten Fasern gegeben. Die Elementaranalysen wurden mit Hilfe von Hewlett-Packard-Analysator 185 durchgeführt.*

$\mathrm{Zu}$ unserer Verfügung stand eine Thermowaage der Firma Mettler Instrumente AG. Damit bestand die Möglichkeit, gleichzeitig TG- und DTA-Messungen durchzuführen. Die Thermogramme wurden mit einem Dreikanal-Schreiber 900 TARKAN der Firma W $+\mathrm{W}$ Electronic Inc. aufgezeichnet.

\section{Bedingungen der Thermoanalyse}

Einwaage:

bei einzelnen Faserproben gegebene Fadenstücke sind $2-3 \mathrm{~mm}$ lang

Atmosphäre: $\quad$ Stickstoff (Strömungsgeschwindigkeit $30 \mathrm{ml} / \mathrm{min}$ )

Temperaturbereich: bis $875^{\circ} \mathrm{C}$

Aufheizraten: $\quad \quad{ }^{\circ} \mathrm{C} / \mathrm{min}$ sind bei einzelnen Thermoanalysen (DTA, TG) gegeben

Auf den Abbildungen stellen die DTA-Kurven die thermischen Prozesse dar (exo- und endothermische), die beim Aufheizen von Chemiefasern vorkommen. Die TG-Kurven stellen den thermischen Abbau der Chemiefasern in Prozenten dar.

\section{DTA:}

Nomex (Einwaage $32,0 \mathrm{mg}$; Aufheizrate $20^{\circ} \mathrm{C} / \mathrm{min}$ )

Zwischen 35 und $150^{\circ}$ breiter endothermer Peak

Entfernung von Feuchtigkeit auf der Faser, Maximum $110^{\circ}$ Zwischen 280 und $380^{\circ}$ mit Maximum bei $332^{\circ}$ kleiner endothermer Peak $380-510^{\circ}$ mit Maximum bei $495^{\circ}$ sehr starker endothermer Peak

Zwischen 440 und $470^{\circ}$ mit Maximum bei $450^{\circ}$ ist ein kleiner exothermer Peak eingelagert

Oberhalb $510^{\circ}$ sehr starker exothermer Peak mit zwei Maxima bei 640 und $760^{\circ}$

Gewichtsverlust (gesamt, \%)

Schmelzpeak, endotherme Zersetzung

Nachkristallisation

exotherme Zersetzung

Nachkondensation

\section{TG:}

$\begin{array}{lrl}25 \text { bis } 280^{\circ} & 4,4 & \text { Entfernung der Feuchtigkeit } \\ \text { Bis } 340^{\circ} & 5,0 & \text { Wasser aus Nachkondensation } \\ \text { Bis } 440^{\circ} & 5,9 & \\ \text { Bis } 560^{\circ} & 30,0 & \text { endothermer Abbau } \\ \text { Bis } 800^{\circ} & 46,6 & \text { exotherme Zersetzung } \\ \text { Bis Endtemperatur } & 58,4 & \text { " }\end{array}$

* Reet Soosalu danken wir für die Durchführung der Elementaranalysen. 

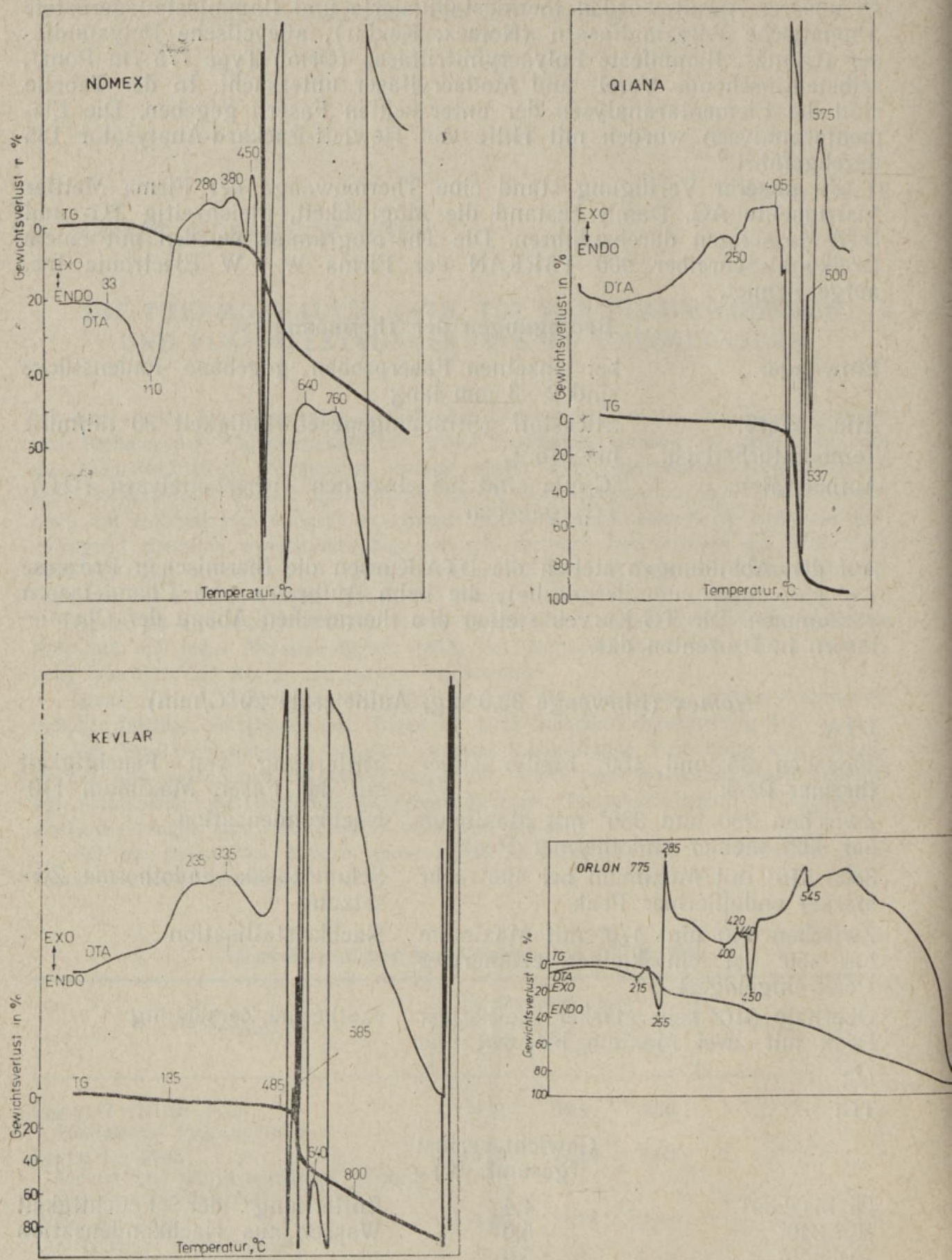

Kevlar (Einwaage 14,2 mg; Aufheizrate $20^{\circ} \mathrm{C} / \mathrm{min}$ )

\section{DTA:}

Zwei kleine endotherme Peaks bei 235 und $335^{\circ}$; oberhalb $475^{\circ}$ starker exothermer Peak mit Maximum bei $585^{\circ}$; kurze Spitze in Richtung endo 
bei $485^{\circ}$ stark ausgeprägter exothermi- setzung scher Effekt

Oberhalb $500^{\circ}$ starke exotherme Reak- Zersetzung (siehe TG) tion

545- $590^{\circ}$ mit Maximum bei $575^{\circ}$ mitt- exotherme vollständige Zersetlerer exothermer Effekt

Oberhalb $590^{\circ}$ geradliniger Verlauf der DTA-Kurve

TG:

25 bis $340^{\circ}$

Gewichtsverlust (gesamt, \%)

Bis $500^{\circ}$

Bis $590^{\circ}$

4,6
95,7
100,0
zung

Bis 590

Orlon Type 775 (Einwaage 27,0 mg; Aufheizrate $5{ }^{\circ} \mathrm{C} / \mathrm{min}$ )

\section{DTA:}

Bis $160^{\circ}$ kleiner exothermer Effekt

Zwischen 160 und $265^{\circ}$ mit einem Maximum bei $255^{\circ}$ starker endothermer Effekt, darin zwischen 215 und $245^{\circ}$ mit Maximum bei $235^{\circ}$ exothermer Effekt

Zwischen 265 und $310^{\circ}$ mit einem Maximum bei $285^{\circ}$ ausgeprägter exothermer Peak

$Z$ wischen 440 und $460^{\circ}$ liegt ein weiterer stark endothermer Effekt mit einem Maximum bei $450^{\circ}$, oberhalb $460^{\circ}$ kleinere endotherme-exotherme Effekte

\section{TG:}

Gewichtsverlust (gesamt, \%)

$\begin{array}{lr}25 \text { bis } 225^{\circ} & 4,6 \\ \text { Bis } 285^{\circ} & 23,1 \\ \text { Bis } 445^{\circ} & 37,0 \\ \text { Bis } 450^{\circ} & 41,7 \\ \text { Bis } 640^{\circ} & 71,3 \\ \text { Bis } 880^{\circ} & 98,5\end{array}$

Entfernung der Feuchtigkeit

Schmelzpeak des Polyacrylnitrils

Kristallisationsbereich

exotherme Zersetzung eines Teils des Polyacrylnitrils (siehe Pyrolyse-Gaschromatographie), Cyclisierung

thermische Zersetzung mit steigender Temperatur

Entfernung der Feuchtigkeit exotherme Zersetzung unter Bildung von $\mathrm{HCN}$ endotherme „Zersetzung

stetiger Abbau mit steigender Temperatur

Verel (PAC $35-80 \%+$ Polyvinylidenchlorid; Einwaage $20 \mathrm{mg}$; Aufheizrate $20^{\circ} \mathrm{C} / \mathrm{min}$ )

\section{DTA:}

Bis $195^{\circ}$ kleine endotherme Reaktion Entfernung der Feuchtigkeit 
$Z$ wischen 195 und $250^{\circ}$ mit einem Maximum bei $244^{\circ}$ endothermer Effekt

Kleiner exothermer Peak bei $225^{\circ}$

Stark ausgeprägter exothermer Peak zwischen 250 und $330^{\circ}$ mit Maximum bei $290^{\circ}$

Zwischen 330 und $550^{\circ}$ mit einem Maximum bei $480^{\circ}$ breiter endothermer Peak

Bei $685^{\circ}$ breiter endothermer Effekt

Oberhalb $685^{\circ}$ Anstieg der DTA-Kurve gegen endotherme Werte

\section{TG:}

Bis $225^{\circ}$ stetige Gewichtsabnahme bis zu $3,0 \%$; oberhalb $225^{\circ}$ starker Sprung in der TG-Kurve; bis $290^{\circ}$ sind infolge der exothermen Reaktion $30,0 \%$ zersetzt, bis $403^{\circ}-37,5 \%$, bis $455^{\circ}-46,3 \%$; anschließend stetiger Gewichtsverlust bis $875^{\circ}$ auf $67,5 \%$. (Anmerkung: Verel verhält sich sehr ähnlich der Modacrylfaser.)

Modacryl (Einwaage 23,1 mg; Aufheizrate $20^{\circ} \mathrm{C} / \mathrm{min}$ )

\section{DTA:}

Bis $210^{\circ}$ praktisch keine Reaktion Kleiner exothermer Effekt bei $240^{\circ}$ Zwischen 210 und $290^{\circ}$ mit Maximum bei $270^{\circ}$ mittlerer endothermer Effekt Oberhalb 290 bis $355^{\circ}$ mit Maximum bei $315^{\circ}$ sehr stark ausgeprägter exothermer Peak

Starker endothermer Effekt zwischen endothermer Abbau 355 und $625^{\circ}$

\section{TG:}

Die Faser ist thermisch sehr stabil, bis $240^{\circ}$ ist lediglich ein Gewichtsverlust von 3,5\% festzustellen, gleichzeitig mit dem Schmelzen beginnt endotherme Zersetzung, die bei $291^{\circ}$ schon $17,5 \%$ ausmacht. Bei $291^{\circ}$ fällt mit der sehr stark exothermen Reaktion ein sprunghafter Gewichtsabfall bis $30,5 \%$ zusammen. Die thermische Stabilität kommt dadurch zum Ausdruck, daß die Faser bis $625^{\circ}$ nur einen Gewichtsverlust bis $42,9 \%$ bei einer stark endothermen Reaktion erleidet. Bis zur Endtemperatur sind nur $64,5 \%$ zersetzt.

Die experimentelle Arbeit wurde am Lehrstuhl der Textilchemic und Makromolekulare Chemie der Technischen Hochschule Aachen, BRD, durchgeführt.

Technische Hochschule Aachen Institut für Chemie

der Akademie der Wissenschaften der Estnischen SSR

Eingegangen am 23. Febr. 1979

\section{I T ERA T UR}

1. Koch, P.A. Faserstofftabellen, Polyamidfasern, Frankfurt/Main, 1976, S. 35. 
O. KIRRET, H. ZAHN, R. HACK

\section{TULEKINDLATE JA TULEKINDLAKS MUUDETUD KEEMILISTE KIUDUDE TERMILINE ANALUOS}

On kirjeldatud kahe termilise analüüsi meetodi - diferentsiaaltermilise analüüsi ja termogravimeetria rakendamist tulekindlate keemiliste kiudude uurimisel. Diferentsiaaltermiline analüüs võimaldab uurida kõrgmolekulaarsete ühendite sulamis- ja kristallisatsiooniprotsesse, polümerisatsiooni- ja polükondensatsioonireaktsioone, termilist lagunemist, dehüdratatsiooni, üleminekut ühest kristallimodifikatsioonist teise ja klaasilisest olekust amorfsesse. Termogravimeetria abil saab iseloomustada kõrgmolekulaarse ühendi keemilist struktuuri, muutusi füüsikalises struktuuris, kvalitatiivset lagunemist olenevalt temperatuurist, vee ja teiste lahustite sorbtsiooni-desorbtsiooni, termilist püsivust, süttimist ja tulekindluse mehhanisme.

O. КИРРET, Х. ЦАН, Р. ХАК

\section{ТЕРМИЧЕСКИИ АНАЛИЗ (ДТА И ТГ) ОГНЕСТОИКИХ И ОГНЕЗАЩИЩЕННЫХ ХИМИЧЕСКИХ ВОЛОКОН}

Дифференциальный термический анализ позволил изучить следующие свойства высокомолекулярных соединеннй (в нашем случае огнестойких и огнезащищенных волокон): процессы плавления и поликонденсации, термическая деградация, дегидратацня, переход из одной фазы кристаллизации в другую, переход из стеклообразной формы в аморфную.

С помощью термогравиметрин (ТГ) охарактеризованы такие свойства высокомолекулярных веществ, как химическая структура, изменения в физической структуре, количественная деградация в зависимости от температуры, сорбция и десорбщия воды и других растворителей, термостойкость, воспламененпе и механизм огнестойкости. 